\title{
ISLAtranscontinental - Integrative Lasertherapie - Mehr als ein Jahrzehnt des Aufbaus
}

\section{Liebe Kolleginnen und Kollegen,}

die Anfänge der Forschung auf dem Gebiet der Lasernadelakupunktur in den Jahren 2002 und 2003 waren turbulent. DerVersuch der ,schmerzfreien Laserstimulation“ an unterschiedlichen Akupunkturpunkten gleichzeitig war neu und gewagt, dessen Ausgang zu Beginn keineswegs Erfolg versprechend. Heute dürfen wir voller Freude auf das bislang Erreichte zurückblicken. Internationale Forschungs- und Behandlungszentren haben sich etabliert. Wichtige Brücken zwischen universitärer Forschung und klinischer Praxis sowie zwischen Ost und West wurden geschlagen. Unsere Zentren in Graz und Göttingen und einige andere in Europa haben sich dabei zu einer Denkfabrik in Bezug auf zukunftsweisende Lasertherapieansätze entwickelt. Unser Dank gilt den Mitgliedern der Gesellschaft und den wissenschaftlichen und industriellen Partnern. Der erfolgreiche Weg im Bereich der integrativen Lasertherapie ist weiter vorgezeichnet, denn neue Laser wie z.B. der gelbe Laser werden bald in Behandlungen der Zukunft zu finden sein. In diesem Sinne: kein Blick zurück, sondern den Fokus voll und ganz auf zukünftige Entwicklungen und Behandlungsstrategien richten ...

Mit herzlichen Grüßen

Michael Weber

Gerhard Litscher
Die ISLAtranscontinental spielt dabei eine wichtige Rolle. Wir arbeiten mit vielen wichtigen Partnern weltweit zusammen und präsentieren uns gemeinsam sehr erfolgreich als wichtige Gesellschaft. In diesem Zusammenhang soll erwähnt werden, dass es neben der Jahresveranstaltung in Beverungen Ende Juni auch wichtige internationale Seminare bzw. Kongresse gibt, wie z.B. die Conference on new approaches in medical laser therapy, die von 16.17. November 2013 in Toronto, Kanada, unter reger internationaler Beteiligung stattgefunden hat. Wie bereits mehrfach angekündigt, wird im nächsten Jahr der erste Weltkongress für High-Tech Akupunktur und integrative Medizin im Mai 2014 in Nanjing stattfinden. ISLAtranscontinental ist Mitveranstalter. Bereits jetzt haben mehr als 20 renommierte SprecherInnen zugesagt. Davon kommen derzeit neun aus China, sieben aus den USA, vier aus Europa (Belgien, Deutschland, Österreich, Russland) sowie jeweils einer aus Kanada und Brasilien.

An dieser Stelle sei uns abschließend erlaubt, allen Kolleginnen und Kollegen ein frohes Weihnachtsfest und ein gesundes und erfolgreiches Jahr 2014 zu wünschen!

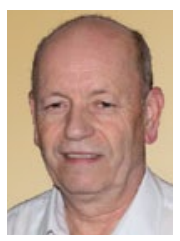

Dr. med. Dipl. Chem. Michael Weber

President für Medical and Clinical Applications der International Society for Medical Laser Applications (ISLAtranscontinental)

Klinik Neu-Mariahilf, Humboldtallee 10-12, D-37073 Göttingen, Tel. +49 5273/367 780

E-Mail:info@isla-laser.org,Internet:www.isla-laser.org

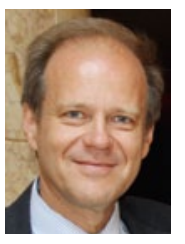

Univ.-Prof. Prof. h.c. DI Dr. techn.

Dr. scient. med. Gerhard Litscher

President for Science and Research der International Society

for Medical Laser Applications (ISLAtranscontinental)

Medizinische Universität Graz, Auenbruggerplatz 29,

A-8036 Graz, Tel: +43 316 385-13907, -83907

E-Mail:gerhard.litscher@medunigraz.at,Internet:http://litscher.info 